\title{
Calibrating a Local Civil Airport Runway Residual Life Prediction Model Using Random Effects and Joint Estimation
}

\author{
Baoli Wei $\mathbb{D}^{1,2}$ Chengchao Guo ${ }^{1}{ }^{3}{ }^{3}$ and Fuming Wang ${ }^{1}$ \\ ${ }^{1}$ School of Water Conservancy and Environment, Zhengzhou University, Zhengzhou 450001, China \\ ${ }^{2}$ School of Civil Engineering and Architecture, Zhengzhou University of Aeronautics, Zhengzhou 450046, China \\ ${ }^{3}$ School of Civil Engineering, Sun Yat-sen University, Zhuhai 519082, China \\ Correspondence should be addressed to Chengchao Guo; guocc@zzu.edu.cn
}

Received 6 May 2020; Revised 4 September 2020; Accepted 20 September 2020; Published 20 October 2020

Academic Editor: Mijia Yang

Copyright (C) 2020 Baoli Wei et al. This is an open access article distributed under the Creative Commons Attribution License, which permits unrestricted use, distribution, and reproduction in any medium, provided the original work is properly cited.

Existing runway residual life prediction models need to be modified using the historical data of evaluated airports during evaluation and management for civil airports in China. If the data measured in the field and the historical data used for model calibration do not represent the actual historical performance of the evaluated airport, the predicted performance of the revised model might be poor. This study used measured pavement performance data for local civil airports in Henan Province from 2007 to 2017. The joint-estimation method was used to establish a functional residual life prediction model for local civil airport pavement with another dataset. A functional residual life prediction model for airport pavement was proposed in consideration of the influence of aircraft traffic and the thickness of the pavement surface layer. Taking into account the differences between samples in the two datasets, nonlinear regression with random-effect analysis and joint estimation were used to explain unobserved heterogeneity at the sample level and heteroscedasticity in the dataset. Based on the results of the established residual life prediction model, the marginal effect of the model parameters and the prediction performance of the entire model were analyzed with the measured data from the local airport pavement. Finally, the engineering applicability of the calibrated prediction model for pavement residual life was further evaluated.

\section{Introduction}

The civil aviation industry is an important strategic industry for China's economic and social development. By the end of 2018, the number of civil aviation transportation airports in China had reached 235 (excluding Hong Kong, Macau, and Taiwan). It is estimated that, by the end of 2020 , the number of civil transport airports in China will reach about 260, and an airport network with reasonable layouts, complete functions, safety, and efficiency will be essentially completed. At the same time, problems related to a lack of balance, coordination, and sustainability remain prominent in the development of the civil aviation industry. With increased air transportation volume and aircraft load, aviation management departments have paid increasing attention to the status of airport pavement. At present, the management teams of large Chinese civil aviation hub airports have begun to develop and use airport pavement management systems to test, evaluate, and maintain the civil engineering facilities of airports [1].

In the 1990s, airport field maintenance work in China met the increasing traffic demand and the requirement for the safe driving of aircraft by building new airport runways or upgrading existing runways. In recent years, with the increase in the service life of Chinese civil airports, runway damage at busy large- and medium-sized airports has become serious. However, due to increases in materials, equipment, and labor costs, as well as increased costs of runway reconstruction, the focus of airport field managers has gradually shifted from the construction of new runways to the maintenance of existing runways. The investment of state and airport authorities in the maintenance of airport infrastructure roads is limited. Therefore, in order to determine how to properly allocate limited funds to the 
maintenance of airport facilities and how to reduce capital investment while meeting maintenance requirements as far as possible, it is necessary to make scientific and reasonable decisions about runway maintenance.

In the evaluation and management of Chinese civil airport pavements, the prediction of the residual life of an airport runway is a relatively important concept. The existing prediction model for the remaining life of a runway needs to be revised using the historical data of the airport being evaluated. If the input and the historical data used for model revisions do not represent actual historical performance data for the evaluated airport, the predicted performance of the revised model may be poor. Therefore, it is clearly required that the prediction of the functional residual life of airport pavements should be based on the actual historical data of airports in different regions in the evaluation code for civil airport pavements in China. The measured data in the field should be used for verification to improve prediction ability after the establishment of the prediction model.

In this study, existing international research on airport pavement performance evaluation was reviewed. Based on existing domestic airport surface performance evaluation methods, the functional residual life prediction model for civil airport pavement was revised using historical data for local airport pavements in Henan Province. To provide support for maintenance decisions for airport runways and provide a reference for the reasonable allocation of limited operation and maintenance funds by airport management departments, a functional residual life prediction model was established for civil airport pavement in consideration of the local characteristics of Henan Province.

\section{Overview of Airport Pavement Performance Monitoring and Evaluation}

2.1. Introduction to Airport Pavement Management System (APMS). Pavement performance evaluation technology is one of the core concepts of pavement management frameworks. The initial research on this topic focused on the evaluation indicators and standards of pavement damage [2, 3], and the "Research Report on Air Force Airport Pavement Maintenance Management System" was completed in 1981. The Pavement Condition Index (PCI) proposed in the report and its set of investigation, calculation, analysis, and evaluation methods have been widely adopted by US military and civil airport pavement management departments when evaluating airport pavement performances. Based on this, the world's first airport pavement management system was developed and named PAVER (now upgraded to MicroPAVER). Since the Construction Engineering Research Laboratory of the US Army (CERL) established the research field of airport pavement evaluation, research on pavement evaluation management technology has been carried out in various countries, and that research has continuously expanded and improved the pavement performance testing and evaluation methods.

The Federal Aviation Administration (FAA) of the US has conducted a series of technical studies to guide pavement test evaluation. Since 2002, the researchers of airport engineering in the US have taken the National Airport Pavement Test Factory [4] (NAPTF) as the main test and trial base, and they have conducted research on airport pavement performance testing, airport pavement design, and maintenance and reconstruction technologies. The domestic pavement evaluation technology in China started late. In 2000, the Airport Division of the Civil Aviation Administration of China (CAAC) issued a manual for civil airfield field maintenance in which the airport pavement investigation and maintenance methods were summarized based on the current technical level of the management of domestic airports. The manual has played a positive role in promoting the development of domestic airport pavement management technology. The first research institution on airport pavement management system in China was Tongji University. In 1995, Tongji University started theoretical research on airport pavement evaluation and management based on Shanghai airport. After 2002, Tongji University developed and established the Shanghai airport pavement management system (SHAPMS) [5] based on experiences with the airport pavement evaluation and management systems in developed countries.

In 2009, the airport department of CAAC, along with Tongji University and the Shanghai airport group, compiled industrial technical standards in the field of civil airport pavement evaluation management in China, that is, the "Technical Specification of Aerodrome Pavement Evaluation and Management" MH/T 5024-2009 [6]. The specification describes in detail the methods for performance evaluation and service life prediction for airport pavement in China. Additionally, the manual specifies that the PCI, SCI (Structural Condition Index), friction coefficient, and International Roughness Index (IRI) of pavement can be used in the performance evaluation of airport pavement. For this evaluation, the specific evaluation method is similar to the MicroPAVER of the FAA, which adopts an empirical regression analysis and pavement design reversal method to analyze PCI in order to predict pavement performance.

\subsection{Review of Airport Pavement Residual Life Prediction} Model. The prediction of airport pavement performance [7] is guided by the theory of road and airport engineering and by forecasting theory. Through a specific method, based on investigation and statistics, with the qualitative analysis and quantitative calculation as the means, by processing airport current and historical data, it has been revealed through analysis that the pavement character and evolution rule in the process of prediction.

Road pavement management systems have been applied earlier in foreign countries and a large amount of road condition data has accumulated. This data is used by national regulatory agencies to establish and develop models applicable to the respective management systems based on the performance of the pavements in their countries or regions. These models include the linear extrapolation model, "S" curve model, and constrained polynomial model [3], among others. The prediction model in the "PAVER" 
pavement management system is the most representative empirical model applied to airport pavement performance prediction. This model is a regression model based on the "pavement family" method, which was established and developed by Shahin et al. based on PCI. [8] In the model, the pavement condition index PCI was used as the predictive index, and a higher-order polynomial was used as the function form whose parameters were estimated with the least-squares method based on the actual data.

Compared with the empirical model, the mechanistic model [9] has a clear theoretical basis and it requires few parameters to be calibrated. In order to make use of the advantages of mechanics and empirical methods, it is a common practice to select the variables and functions in empirical models with mechanical methods and to revise or calibrate the mechanical models through a large number of laboratory and outdoor experiments or empirical regression methods, that is, the mechanistic-empirical model. In recent years, more and more scholars have been inclined towards the mechanistic-empirical model. However, the mechanistic-empirical model inherited the shortcomings of the mechanical model. For example, the calculation process is complicated and the workload is large, only the mechanical index or the material performance index (e.g., stress, strain, deflection, and modulus) model can be established, and it is difficult to update the model. In 1995, the FAA proposed a pavement design method [10] for a new generation of large aircraft based on the cumulative damage principle, elastic layer system theory, and pass-to-coverage ratio, and the reverse of the design procedure method was taken as the prediction model for the remaining life of a pavement structure. This model was a typical representative of an airport pavement mechanistic-empirical model. Based on a method that was the reverse of the design procedure in this model, Tongji University established a prediction model for the remaining life of rigid pavement structures of airports [11] based on the design specifications of airport pavement in China. Then, the model was applied and verified in the pavement structural evaluation of several civil airports in China.

At present, many PMS software programs have developed a prediction model for pavement performance using a probabilistic model. Probabilistic prediction models take into account the probability associated with future pavement performance as a random variable, hence predicting the pavement condition at a certain probability level based on engineering judgment or analyses of historical data. Probabilistic prediction models include the residual curve probabilistic prediction model [12], Bayesian probabilistic prediction model [13], Semi-Markov probabilistic prediction model [14], and Markov probabilistic prediction model [15], among which the Markov process model [16-22] is the most widely applied and the most perfect.

In recent years, more and more intelligent algorithms have been used to predict pavement performance. These intelligent models mainly include the Artificial Neural Network, genetic algorithms, gray theory models, and modern time series models. Among these models, the gray prediction model has been widely applied in China, for example, Liu et al. [23-25] applied the gray theory model to predict pavement performance, which has rarely been applied in other countries. However, modern time series methods are rarely used in China, while relatively more of them are applied in other countries. For example, Ben-Akiva et al. [26-28] applied modern time series methods to predict pavement performance.

In pavement performance evaluation and remaining life estimation, PCI is generally used as the evaluation index of pavement damage in most airports in the world. Some Chinese scholars have also proposed the use of the broken slab ratio (for cement) and the cracking ratio (for asphalt) as additional evaluation indexes for pavement damage. With the continuous development of the research field of airport pavement evaluation, people have found that the service characteristics of airport pavement cannot be fully represented using only the surface condition of pavement. Therefore, people have begun to pay attention to the structural condition and structural capacities of airport pavement and to take these as one of the main concepts of pavement performance evaluation. At the same time, more evaluation indexes have been introduced in the evaluation of airport pavement performance, such as skid resistance, roughness, and drainage capacity. Hence, the evaluation theory of airport pavement performance has kept being expanded and improved upon, and a relatively complete pavement evaluation system has gradually formed.

\section{Calibration Methods and Prediction Analysis Techniques}

3.1. Random-Effect Analysis Model. The random-effect analysis model can flexibly and effectively combine information from different sources and explain different sources of error, so it is especially suitable for the study of panel data [29]. The mixed effects of the model include fixed effects and random effects. When an interindividual variation cannot be fully explained by the fixed effects in the model, the random effects within an individual level are introduced to characterize this additional difference in the sample. The difference between a fixed-effect model and a random-effect model alone relies on whether it is desirable to make inferences about the particular levels of the classification factor defined in the experiment or to make an inference about the experimental units from which the levels are drawn. According to the types of response variables in the models, random-effect analysis models can be divided into linear mixed-effect models and nonlinear mixed-effect models.

The deterioration characteristics of pavement with the same structural composition are similar, while pavements with the same function and similar environmental conditions with the same structure type are more similar. This constitutes a two-level, three-level, or even multilevel data structure (that is, Panel Data). In the research on pavement performance prediction, more is paid attention to the overall deterioration features of pavement performance affected by various factors rather than the performance change characteristics of an individual pavement area. This inevitably involves the processing and analysis of multilevel 
heterogeneous observation data. Traditional models have no optionality, and random-effect models are optimal for these analyses.

The random-effect model is used to combine both types of effects within one model, with fixed-effect parameters explaining the behavior of the population means between a given set of treatments (intraindividual variation) and the random effects representing the variability among testing units (interindividual variation). Therefore, the randomeffect model can quantitatively analyze the source of variation of pavement performance observation data, and it can more reasonably be used for group pavement.

A simple model is used to illustrate the mixed-effect framework. The linear mixed-effect model [30] includes two level 1 explanatory variables $x_{1 i j}$ and $z_{1 i j}$ and one level 2 explanatory variable $w_{1 j}$. A dependent variable $y$ is modeled as a simple linear function of a covariate $x$ as follows:

$$
\begin{aligned}
& y_{i j}=\beta_{0 j}+\alpha_{1} x_{1 i j}+\beta_{1 j} z_{1 i j}+e_{i j}, \\
& \beta_{0 j}=\gamma_{00}+\gamma_{01} w_{1 j}+u_{0 j}, \\
& \beta_{1 j}=\gamma_{10}+\gamma_{11} w_{1 i j}+u_{1 j},
\end{aligned}
$$

where $y_{i j}$ is the $j$ th observation of the dependent variable for the experimental unit $i . \beta_{0 j}$ and $\beta_{1 j}$ are the fixed-effect parameters related to the entire population. $u_{0 j}$ and $u_{1 j}$ are random-effect parameters associated with unit $j$. For unit $j$, these represent deviations from the population parameters $\beta_{0 j}$ and $\beta_{1 j}$, respectively. $e_{i j}$ is the error term for observation $j$ on unit $i$; it is normally assumed to be distributed as $N\left(0, \sigma^{2}\right)$, where $\sigma^{2}$ is the variance of $e_{i j}$. It is also commonly assumed that these errors are independent and that they have constant variance (all these hypotheses need to be corrected if not satisfied). $\alpha_{1}$ and $\gamma_{i j}$ are the regression coefficients of the level 1 and level 2 variables.

Equation (1) is a level 1 equation for analyzing $y_{i j}$ variation. The subscript " $j$ " in the level 1 intercept $\left(\beta_{0 j}\right)$ indicates that the level 1 intercept changes across level 2 units. The effect of the level 1 variable $x_{1 i j}$ on $y_{i j}$ does not vary across level 2 units, but the effect of the variable $z_{1 i j}$ on $y_{i j}$ varies with level 2 units. That is, there is a level 1 fixed slope $\alpha_{1}$ and a level 1 random slope $\beta_{1 j}$. Corresponding to the level 1 random regression coefficients $\beta_{0 j}$ and $\beta_{1 j}$ are two level 2 equations, namely, equations (2) and (3). In these equations, the level 1 random regression coefficient becomes the dependent variable (or response variable).

For the statistical analysis of nonlinear repeated measurement data, the method of the approximation regression model of a nonlinear function is commonly used to solve the nonlinear problem. There is autocorrelation between the nonlinear repeated observation data and the random variance can be divided into at least two levels, that is, interindividual variance and intraindividual repeated measurement variance. This model is also called the hierarchical nonlinear model. The nonlinear mixed-effect model can not only identify and estimate the variation between and within individuals but also take into account the nonlinear relationship between explanatory variables and response variable parameters, allowing fixed effects and random effects to enter the nonlinear part of the model. The probability density functions of the response variables in the nonlinear mixed-effect model can be normal, binomial, or Poisson.

The performance deterioration equation of road pavements (or other pavements) around the world has been comprehensively considered, and the performance deterioration equation of airport pavements in MH/T 5024-2009 was combined in this study. The specific form is shown in equation (4). This equation could accurately reflect the entire process of pavement performance deterioration and fit various deterioration models. Theoretically, the performance index (including PCI or Riding Quality Index (RQI)) should have been reduced monotonically with the increase of the service life or the cumulative axial load times. Equation (4) met the necessary boundary conditions. The equation was simple in form, and the parameter definition was clear, so the equation could provide a basis for the prediction of the functional residual life of airport pavement:

$$
Y=Y_{0} \cdot\left(1-e^{-(\alpha / t)^{\beta}}\right)
$$

where $Y$ is the pavement performance index (which can be PCI, RQI, or IRI). $Y_{0}$ is the value of the pavement performance index at a certain time. Generally, it is the initial value of a pavement performance index when the pavement is put into use. $t$ is the service time of the pavement. $\alpha$ and $\beta$ are the model parameters of the deterioration equation, which are related to the individual characteristic variables.

\subsection{Joint-Estimation Methods for Multiple Data Sources.} The joint-estimation method is a preferred statistical method when using multiple data sources for parameter estimation because joint estimation can determine not only the explanatory variables common to all data sources but also the explanatory variables unique to each data source [31]. The joint-estimation method was first used by Morikawa et al. [32] to model travel demand. Archilla and Madanat [33] and Prozzi and Madanat [34] used the joint-estimation method to build a pavement performance deterioration model.

To illustrate the joint-estimation method, in this study, it was assumed that two different data sources A and B were considered. Both databases contained the same dependent variable PCI (which was also the object of this project) and several influencing factors (explanatory variables) such as the frequency of aircraft departure and landing and the thickness of the pavement surface. Specifically, each of these datasets could use the following expression to represent each model:

$$
\begin{aligned}
& E\left(\mathrm{PCI}^{\mathrm{A}} \mid x^{\mathrm{A}}, w\right)=g^{\mathrm{A}}\left(\beta^{\mathrm{A}}, x^{\mathrm{A}}, \alpha, w\right), \\
& E\left(\mathrm{PCI}^{\mathrm{B}} \mid x^{\mathrm{B}}, z\right)=g^{\mathrm{B}}\left(\beta^{\mathrm{B}}, x^{\mathrm{B}}, \gamma, z\right),
\end{aligned}
$$

where $E\left(\mathrm{PCI}^{*} \mid \cdot\right)$ is the conditional expectation function of PCI with the functional forms $g^{\mathrm{A}}(\cdot)$ and $g^{\mathrm{B}}(\cdot)$ for Models A and $\mathrm{B}$, respectively. $\mathrm{PCI}^{\mathrm{A}}$ and $\mathrm{PCI}^{\mathrm{B}}$ represent Models $\mathrm{A}$ and 
$\mathrm{B}$ of the PCI, $x^{\mathrm{A}}$ and $x^{\mathrm{B}}$ are vectors of the explanatory variables shared by Models $\mathrm{A}$ and $\mathrm{B}$, and $w$ is the vector of the explanatory variables unique to Model A and dataset A. $z$ is the vector of the explanatory variables unique to Model $\mathrm{B}$ and dataset B, $\alpha$ and $\gamma$ are the vectors of the model parameters related to $w$ and $z$, respectively, and $\beta^{\mathrm{A}}$ and $\beta^{\mathrm{B}}$ are vectors of the model parameters related to $x^{\mathrm{A}}$ and $x^{\mathrm{B}}$, respectively.

Depending on the explanatory variables, different scenarios are possible. Only the most common scenario was considered in this study, for which both datasets shared some explanatory variables and had some variables with predefined levels for each source. In this case, some of the estimated parameters were common to both datasets and others were unique to a dataset. The potential advantages of this scenario were that it allowed the identification of explanatory variables that might not have been available in some datasets, and it permitted determination of the bias parameters to account for the different marginal effects estimated for the explanatory variables in common between datasets. In terms of the models shown previously, the values of the set of common parameters might have been different, that is, $\beta^{\mathrm{A}} \neq \beta^{\mathrm{B}}$. Therefore, the relationship between the $i$ th element of the set of parameters of each model could be expressed as $\beta_{i}{ }^{\mathrm{A}}=\beta_{i}^{\mathrm{B}}+\delta_{i}$ or $\beta_{i}{ }^{\mathrm{A}}=\mu_{i} \beta_{i}{ }^{\mathrm{B}}$, where $\delta_{i}$ and $\mu_{i}$ are bias parameters or bias correction coefficients. This feature was called bias correction and it was one of the main advantages of joint estimation. Two other advantages were identification and statistical efficiency. Identification relates to the complementary characteristics of the datasets. For example, one dataset might have contained explanatory variables with no variability in other datasets, thus allowing the identification of the effects of these variables from a single dataset. In this study, this was different from a missing relevant explanatory variable (which would have led to biases). Instead, these variables were not really missing but rather had a predefined single level in some datasets. Statistical efficiency was achieved when a parameter estimate common to all datasets was determined using all available data. Because of the larger sample size, this resulted in lower variance of the parameter estimate. In this study, the jointestimation method was used for two historical datasets containing different pavement structural types, aircraft traffic, and test conditions. The detailed analysis is discussed in Section 4.

\section{Airport Pavement Data Preparation}

The historical data of an airport is the basis for establishing a model for estimating the remaining life of airport pavement. In the past two decades, China has accumulated pavement test data for the PCI for more than 60 civil airports. On the one hand, this data can represent the pavement surface of Chinese civil airports. On the other hand, the data can also represent the typical characteristics of airport pavement performance field test data. In this study, two history datasets of airport pavement tests with different characteristics were used as data sources for analysis. The first dataset was the field test history dataset for PCI on domestic airport pavement, and the second dataset was the field test dataset for Henan local airport runways from 2007 to 2017. The on-site detection is shown in Figure 1. The characteristics and details of the first dataset can be found in [35]. Only the second dataset is briefly introduced here.

The second dataset was the time series data of the runway and taxiway pavement of a southern flight area in 2007, 2013, and 2017 at a local airport in Henan. The south runway of the airport was $3,400 \mathrm{~m}$ long and $45 \mathrm{~m}$ wide, while the north runway was $3600 \mathrm{~m}$ long and $60 \mathrm{~m}$ wide. This was the Chinese trunk transport airport and a national first-class aviation port. In 2016, the passenger throughput of the airport ranked the 15th among civil airports in China. The airport was opened to traffic in 1997, and its south flight area has been used for 22 years. With the continuous increase of air traffic at the airport, the south airfield was approaching its designed service life at the time of the study.

To comprehensively grasp the situation of the pavement in the southern flight area and provide a basis for the management and renovation plan of the flight area, the airport management department developed a detailed inspection plan. In 2007, 2013, and 2017, the airport management department carried out comprehensive tests on runways, taxiways, and contact surfaces in the south flight area of the airport, and the department conducted comprehensive analyses of the field test data to form a high-value database. The main content of the database included the history of pavement maintenance and rehabilitation (M\&R), aviation portfolios, and the combination of aircraft types, the degradation of the pavement condition index PCI, pavement roughness IRI, pavement surface sliding resistance coefficient, pavement deflection value, structural capacity of pavement, joint load transfer, and condition of the pavement void, pavement radar detecting data, and strength of the concrete pavement.

In order to avoid new errors in the process of data preprocessing, this research was based on the characteristics of data sources and data processing methods in the literature [36]. The second dataset was divided into groups consisting of pavement areas that had the same amount of average annual aircraft traffic and the same pavement structures. It was assumed that the PCI in the same group was homogeneous. Due to the different observation time periods of individual pavement, the time series data in the group could be extended by a "time lapse." At the same time, the individual data that was special or doubtful was eliminated. Finally, 91 sets of rigid pavement data were obtained. Some of this data is shown in Table 1.

In Table 1, the age represents the number of years since the airport was built and operation was started. The region was divided into a runway area and a taxiway. The runway was further divided into a section with a concrete thickness of $38 \mathrm{~cm}$ and a section with a concrete thickness of $34 \mathrm{~cm}$. The average annual aircraft traffic [6] was the average cumulative number of equivalent main aircraft type operations in the airport in the test year. The thickness was the design thickness of the concrete surface layer of the airport pavement. 


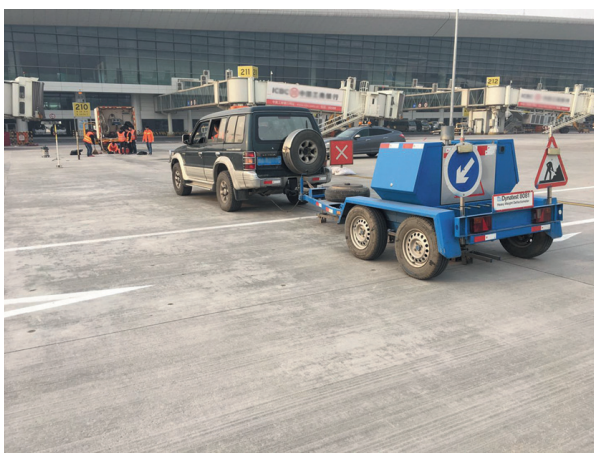

(a)

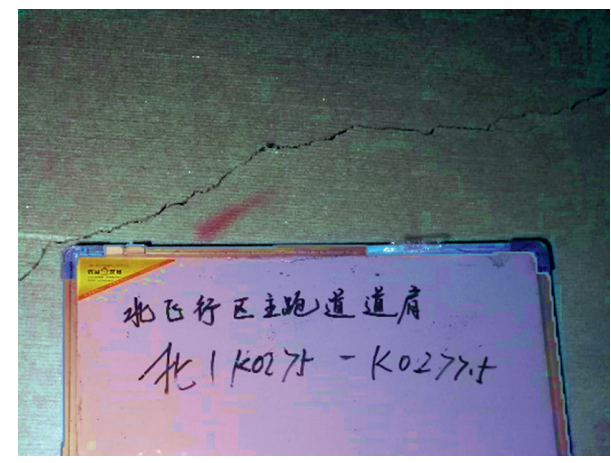

(b)

FIGURE 1: Field inspection of airport runway: (a) inspection equipment; (b) crack detection.

Table 1: Part of the second dataset.

\begin{tabular}{ccccccc}
\hline Group ID & Age (years) & PCI & Runway or taxiway & Region & Average annual aircraft traffic & Thickness $(\mathrm{cm})$ \\
\hline \multirow{4}{*}{17} & 1 & 99 & Runway & Runway $38 \mathrm{~cm}$ thick & 74325 & 74325 \\
& 15 & 81 & Runway & Runway $38 \mathrm{~cm}$ thick & 74325 & 38 \\
\hline \multirow{4}{*}{50} & 19 & 76 & Runway & Runway $38 \mathrm{~cm}$ thick & 74325 & 38 \\
& 1 & 99 & Runway & Runway $34 \mathrm{~cm}$ thick & 74325 & 34 \\
& 15 & 80 & Runway & Runway $34 \mathrm{~cm}$ thick & 74325 & 34 \\
76 & 19 & 73 & Runway & Runway $34 \mathrm{~cm}$ thick & 74325 & 38 \\
& 1 & 99 & Taxiway & Taxiway $38 \mathrm{~cm}$ thick & 74325 & 38 \\
\hline
\end{tabular}

\section{Specification of Random-Effect Nonlinear Mixed Model}

The curve shape of the function expressed by equation (4) was mainly controlled by the parameters $\alpha$ and $\beta$, but $\beta$ was more sensitive to the influence of the curve shape than $\alpha$. When the combination of $\alpha$ and $\beta$ was appropriate, the shape of the curve could be close to a straight line. Therefore, there was a one-toone relationship between any complex pavement performance curve and $\alpha$ and $\beta$, so a two-dimensional point $(\alpha, \beta)$ could be used to describe the decay process of the pavement performance and the development rules of the performance quantitative analysis. At the same time, an important inference could be drawn, that is, $\alpha$ and $\beta$ could be represented as functions of the factors that affected $\alpha$ or $\beta$. For example, $\alpha=f$ (axle load, structural strength, surface layer thickness, base layer type, environmental conditions, and material type), and $\beta=f$ (axle load, structural strength, surface layer thickness, base layer type, environmental conditions, and material type), where $f(\cdot)$ is an arbitrary function.

As mentioned above, the expected function of the model determined by equation (7) was taken as the objective function. Equation (7) is the transformation form of equation (4). Considering the characteristics of the two datasets, the joint-estimation method and the random-effect model were used to determine the mixed model for predicting the remaining life of the airport pavement. The following sections present detailed steps for the aforementioned mixed model:

$$
\mathrm{PCI}_{t}=\mathrm{PCI}_{t 0}-100 \cdot e^{-(\alpha / t)^{\beta}}+\gamma_{i j}
$$

where $\mathrm{PCI}_{t}$ is the PCI of the pavement at time $t$ and $\mathrm{PCI}_{t 0}$ is the PCI of the pavement at the initial time $t_{0}$. Its value was related to the structural type of the airport pavement, the amount of aircraft traffic, and other influencing factors, and the value was generally within the range of 90 100. $\gamma_{i j}$ is the random normal variance; the meanings of other parameters are the same as those of equation (4).

Because all of the factors affecting the performance of the pavement surface also affected the parameters $\alpha$ and $\beta$, the individual model measurement parameters were combined through an exploratory analysis, and the logarithmic transformation of $\alpha$ and $\beta$ was converted into a linear expression in order to obtain the group structure model as follows:

$$
\left\{\begin{array}{l}
\log \alpha_{i}=\log a_{1}+a_{2} \log N+a_{3} \log H+\log b_{0 i} \\
=k_{1}+k_{2} \log N+k_{3} \log H+u_{0 i}, \\
\log \beta_{i}=\log c_{1}+c_{2} \log N+c_{3} \log H+\log b_{1 i} \\
=m_{1}+m_{2} \log N+m_{3} \log H+u_{1 i} \\
u_{j i} \sim N\left(0, \sigma^{2} D\right)
\end{array}\right.
$$

where $N$ is the aircraft traffic volume, $H$ is the pavement surface thickness $(\mathrm{cm})$, the individual model parameters $\alpha_{i}$ and $\beta_{i}$ are normal random variables, where $a$ and $c$ are fixed effect parameters, and $b_{j i}$ is a random-effect term.

In order to improve the prediction efficiency, a randomeffect method was used to establish a prediction model. The 
random-effect mixed model combined two types of effect factors within one model, with the fixed effects explaining the behavior of the population means between a given set of treatments (intraindividual variation) and the random effects representing the variability among testing units (interindividual variation). Then, the source of variation of the pavement performance observation data could be quantitatively analyzed with the mixed-effect model. This model could present the deviation of each individual from the population mean and the unobserved heterogeneity in the population. The nonlinear random-effect mixed model was obtained through random effects, and the parameters used in the model were the parameters as those in equation (8).

According to the joint-estimation method described in Section 3.2, the dummy variable DS was further introduced to represent different data sources, that is, when the data belonged to dataset 1 , the value of DS was 0 ; when the data belonged to dataset 2, the value of DS was 1 .

After exploratory analysis, the model parameters were calibrated by random effects, all of the parameters that were not statistically significant (significance level of 0.05 ) were removed, and the significance of the other parameters was re-evaluated. Finally, the random-effect nonlinear mixed model was obtained:

$$
\left\{\begin{array}{l}
\mathrm{PCI}_{t}=\mathrm{PCI}_{t 0}-100 \cdot e^{-(\alpha / t)^{\beta},} \\
\mathrm{PCI}_{t 0}=c_{1}, \\
\log \alpha=k_{1}+\left(k_{2}+\mu_{2} \mathrm{DS}\right) \log N+\left(k_{3}+\mu_{3} \mathrm{DS}\right) \log H+u_{0 i}, \\
\log \beta=m_{1}+\left(m_{2}+\lambda_{2} \mathrm{DS}\right) \log N+\left(m_{3}+\lambda_{3} \mathrm{DS}\right) \log H+u_{1 i},
\end{array}\right.
$$

where $c_{i}, k_{i}$, and $m_{i}$ are the fixed effect coefficients of the model, $u_{j i}$ is the normal random variance of the $j$ th individual in the $i$ th population, and $\mu_{i}$ and $\lambda_{i}$ are the bias correction coefficients for the joint estimation.

\section{Results and Discussion}

After the aforementioned modeling exploration, a "final" model could be established. However, a model with good fit data (such as -2LL (-2 log likelihood) or less bias, with a smaller Akaike Information Criterion (AIC) and a smaller Bayesian Information Criterion (BIC)) [30] was not necessarily a satisfactory model. As emphasized in statistics, model selection is a process driven by statistics and research theory. The purpose of the modeling was not only to find a satisfactory statistical model for data fitting but also to establish a simple model with interpretable results.

\subsection{Estimation of the Nonlinear Mixed Model. The param-} eters of the residual life prediction model with equation (9) were estimated using the random-effect approach, taking into account the joint-estimation method. The estimated parameters and the asymptotic statistics are given in Table 2 .

It can be seen from Table 2 that the estimated variance of the random-effect mixed model when using the joint-estimation approach and including the explanatory variables $\log N$ and $\log H$ was $\sigma^{2}=1.565$. Compared with the nonlinear model (in the exploratory modeling, the nonlinear least-squares method was used to establish the nonlinear fixed-effect model, and the estimated variance was 3.228), the coefficient was reduced by half, and the reduction proportion was nearly $50 \%$. This indicated that the random-effect method played a significant role in improving the fitting efficiency of the model and this method could better explain the heteroscedasticity of the population and the intergroup effect.

In order to further illustrate the overall superiority of the model, the following analysis process was conducted. In the process of modeling using the random-effect nonlinear mixed model, the results of all levels of nesting used in the model could be displayed. The default was to display the intergroup fitting value, which included random effects and fixed effects. This model was called the mixed-effect model in this study. By changing the random-effect term, only the population fitting value could be displayed, that is, only the fixed effect could be considered without the random effect. This model was called the no-mixed-effect model in this study. To illustrate the difference between the models with and without random effects, a comparison of the fit values and the predicted values was made. The results are shown in Figure 2(a) (no-mixed-effect) and Figure 2(b) (mixedeffect).

It can be seen from Figure 2 that the data points were generally clustered at the diagonal of $45^{\circ}$, indicating that the nonlinear model could fit the data set well regardless of whether random effects were taken into account. By comparing Figures 2(a) and 2(b), it could be concluded that for dataset 2, the horizontal line pattern of the data point aggregation in Figure 2(a) no longer existed in Figure 2(b) (the data distribution is more uniform along the $Y$-axis). As expected, the model with mixed effects interpreted the data much better than the model with only fixed effects. This was because when using the mixed-effect approach, each individual in each population predicted by the model contained two factors of the random effects, one of which was $u_{0 i}$ in the $\alpha$ parameter and the other of which was $u_{1 i}$ in the $\beta$ parameter. In other words, the error of each individual could be explained with two independent random errors, so the mixed-effect model was significantly better than the no-mixed-effect model.

6.2. Validity of Assumptions of Regression for Random-Effect Nonlinear Mixed Model. The normality assumption for the standardized residuals could be validated using box-andwhiskers plots. A box plot of the standardized residuals for all of the individuals of the mixed-effect model is shown in Figure 3. In the figure, the black marker points represent the median of the within-specimen residuals. The samples 1-16 comprised dataset 1 , and the other samples comprised dataset 2. In the figure, the two datasets are demarcated by the red vertical line. As can be seen from Figure 3, the median distribution of the sample residual box line of the test samples was larger in proportion to the value of 0 . A small number of zero values were not included in the sample residual range (i.e., box line length) in the two datasets. This showed that, by allowing for random effects in the model, it was possible to capture the deviations from the overall mean 
TABle 2: Parameter estimation $n$ for random-effect nonlinear mixed model.

\begin{tabular}{|c|c|c|c|c|c|}
\hline & Estimate & Standard error & $T$-statistic & $P$ value & Annotation \\
\hline \multicolumn{6}{|c|}{ Fix-effect parameter } \\
\hline$k_{1}$ & $1.13 E+00$ & $1.22 E-01$ & 9.28 & $<0.0001$ & Intercept \\
\hline$k_{2}$ & $-8.37 E-02$ & $1.12 E-02$ & -7.51 & $<0.0001$ & Gradient \\
\hline$k_{3}$ & $3.46 E-01$ & $1.05 E-01$ & 3.30 & 0.0013 & Gradient \\
\hline$m_{1}$ & $-1.10 E+00$ & $3.36 E-01$ & -3.26 & 0.0015 & Intercept \\
\hline$m_{2}$ & $-1.05 E-01$ & $2.88 E-02$ & -3.65 & 0.0004 & Gradient \\
\hline$m_{3}$ & $1.11 E+00$ & $2.83 E-01$ & 3.93 & 0.0002 & Gradient \\
\hline$c_{1}$ & $9.89 E-01$ & $1.19 E-03$ & 833.55 & $<0.0001$ & Intercept \\
\hline$\mu_{2}$ & $-2.70 E-01$ & $1.85 E-01$ & -1.46 & 0.1476 & Bias coefficient \\
\hline$\mu_{3}$ & $1.04 E+00$ & $5.81 E-01$ & 1.79 & 0.0767 & Bias coefficient \\
\hline$\lambda_{2}$ & $5.22 E-01$ & $2.55 E-01$ & 2.05 & 0.0431 & Bias coefficient \\
\hline$\lambda_{3}$ & $-1.84 E+00$ & $8.00 E-01$ & -2.30 & 0.0234 & Bias coefficient \\
\hline \multicolumn{6}{|c|}{ Random-effect parameter } \\
\hline$u_{0}$ & $2.21 E-04$ & $1.53 E-04$ & 1.45 & 0.1508 & Variance \\
\hline$u_{1}$ & 1.' $E-03$ & $3.86 E-04$ & 3.28 & 0.0014 & Variance \\
\hline$\sigma^{2}$ & $1.57 E+00$ & $1.52 E-01$ & 10.33 & $<0.0001$ & Variance \\
\hline
\end{tabular}

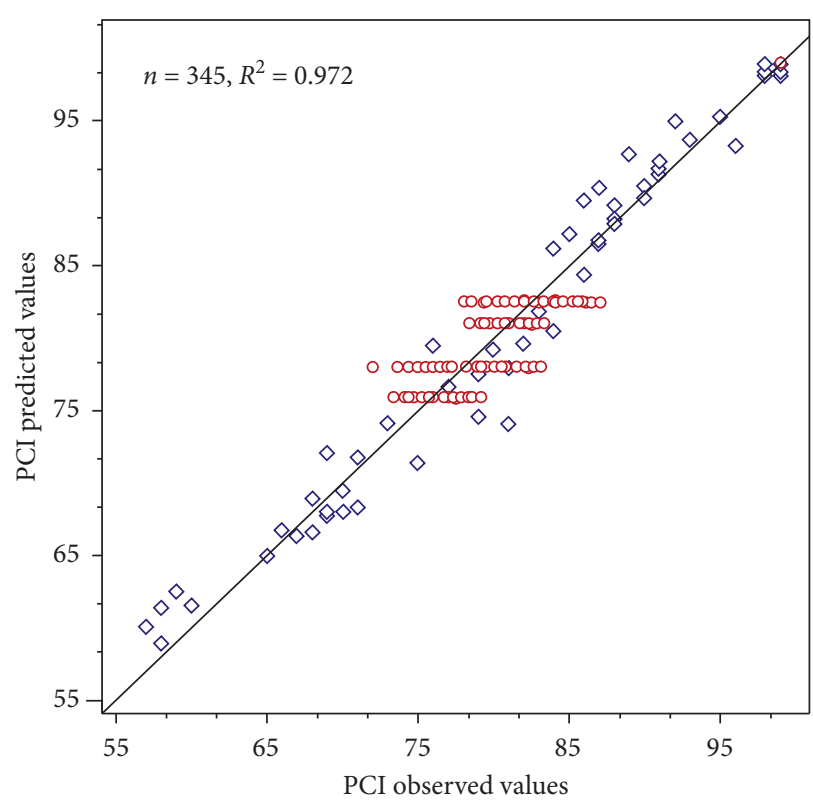

Dataset

$\diamond 1$

$\circ 2$

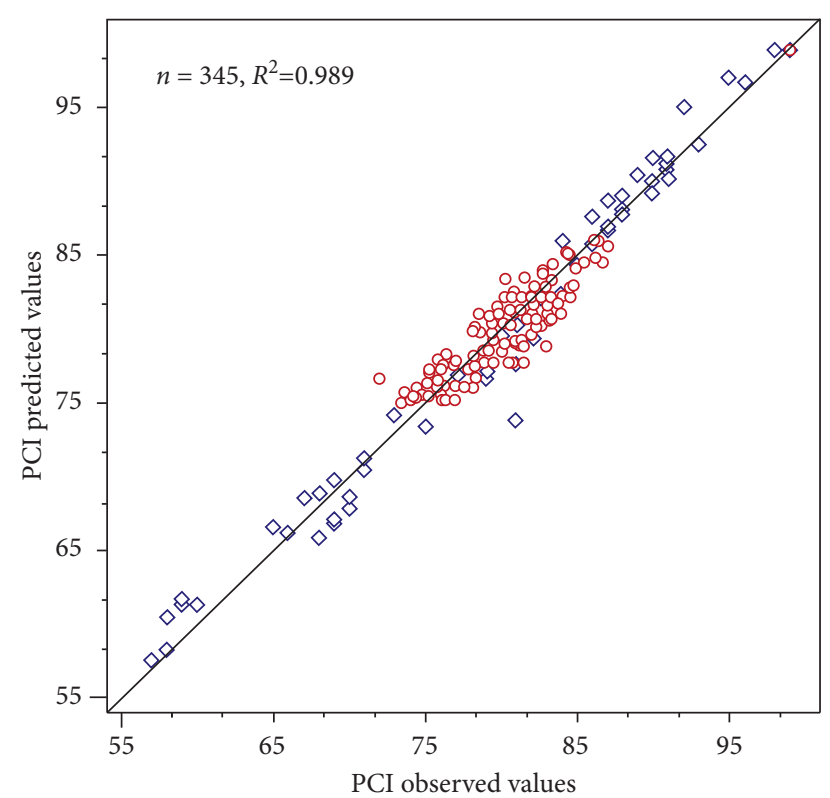

Dataset

$\diamond 1$

$\circ 2$

(a)

(b)

FIGURE 2: Observed and predicted values of PCI in the mixed model: (a) no-mixed-effect $\left(n=345, R^{2}=0.972\right)$; (b) mixed-effect $(n=345$, $\left.R^{2}=0.989\right)$.

and thus improve the modeling of the data generation process. It also showed that the random effects could capture unobserved heterogeneities between specimens and eliminate them from the error term.

In order to verify the parameter estimation of the random-effect nonlinear mixed model, it was assumed that the variance conformed to the normal distribution, so the two graphs of the normality assumption for the standardized residuals and the random effects were plotted (due to space limitations, the two graph are not displayed.)

The following conclusions could be drawn from the figures. The assumption of the normality of the random effects made for the model estimation was also empirically verified. The hypothesis test of all of the parameter estimates in the mixed-effect model provided more reliability than the nonlinear least-squares fit model. The assumption of the normality of the sample residuals for the mixed-effect model was verified.

6.3. Evaluation of the Prediction Performance of the Revised Model. This section describes the assessment of the model in terms of its reasonableness in order to account for several characteristics. Several figures are presented, 


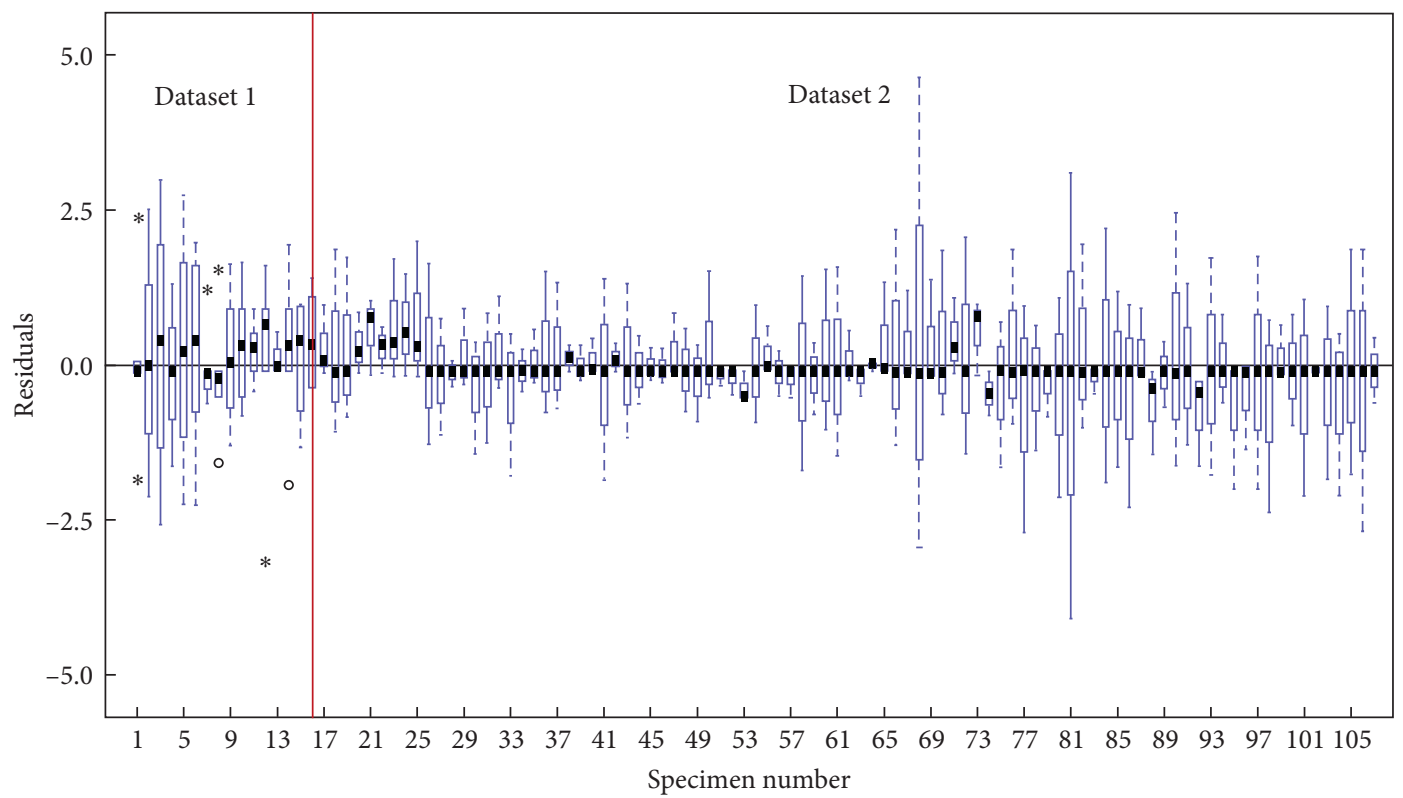

FIGURE 3: Box plot of mixed model residuals (classified by dataset).

showing how the model approached the actual measured values. Other plots are shown to illustrate the sensitivity of the model predictions when certain variables were varied. In order to avoid the randomness of sample selection, when presenting the graph for each sample in each group, a sample with a large residual that contained the characteristics of the parameter to be analyzed was selected in the dataset. For example, when analyzing the influence of aircraft traffic on the prediction performance of the model, the samples $1 / 3 / 4$ with large residuals between the predicted PCI and the measured PCI with different aircraft traffic in dataset 1 were selected. In addition, it should be noted that the symbol "s $14-\lg N=3.62$ " in Figure 4(a) represents the PCI data of the 14th group of airport pavement with $\log N=3.62$ (the logarithm of the aircraft traffic). "S116-H15 cm" represents the PCI data of the 116th group of pavement with a surface concrete thickness of $15 \mathrm{~cm}$. The other symbols have similar meanings.

6.3.1. Prediction of the Model with Varying Flight Traffic. In order to evaluate the prediction performance of the random-effect mixed model for the pavement performance of different traffic levels, samples with the same pavement thickness but different traffic levels were selected for analysis. Specifically, sample S14 $(\log N=3.62)$ and sample S9 in data set 1 were selected for analysis, along with $(\log N=4.29)$, sample S15 $(\log N=4.42)$, and sample S2 $(\log N=4.56)$. The thickness of the concrete pavements of these individual samples was $32 \mathrm{~cm}$. The PCI prediction curve is shown in Figure 4(a).

As can be seen from Figure 4(a), when the service life of the pavement $(t)$ was less than 10 years, the PCI prediction curve of the mixed-effect model with different aircraft traffic levels was generally similar, and the difference was not obvious. However, after $t>10$ years, the PCI prediction curve with different aircraft traffic levels showed significant differences. With the increase of the aircraft traffic, the value of the PCI decreased, and the higher the level of aircraft traffic was, the greater the degraded rate of the PCI was. This result indicated that, with the increase of aircraft departure and landing frequencies, the deterioration rate of the airport pavement surface layer also increased, leading to the decrease of the PCI value. This also proved that the mixed-effect model could capture the PCI deterioration trend of individuals with different aircraft traffic levels (limited to the aircraft traffic range included in the dataset).

It is worth noting from Figure 4(a) that, in the initial service years of the airport pavement (about 10 years as shown in the graph), due to the structure or material characteristics of the airport pavement itself, the aircraft traffic had little impact on the pavement deterioration. However, when the service time of the airport pavement was longer than a certain number of years, the aircraft traffic had a significant impact on the pavement deterioration. This also showed that the aircraft load had a certain cumulative effect on the damage of the airport pavement. The determination of whether this effect was linear cumulative or nonlinear cumulative requires further study.

In addition, it was noted that the predicted curves of the aircraft traffic volume $\log N=4.29$ and $\log N=4.42$ generally coincided with each other (with the other parameters being the same), which indicated that the performance deterioration trend of the airport pavement at these two traffic volume levels was essentially the same. However, the prediction curves of the aircraft traffic volume $\log N=4.42$ and $\log N=4.56$ were significantly different (with the other parameters being the same), which indicated that the performance deterioration trend of the airport pavement at the two traffic volume levels was significantly different. The 


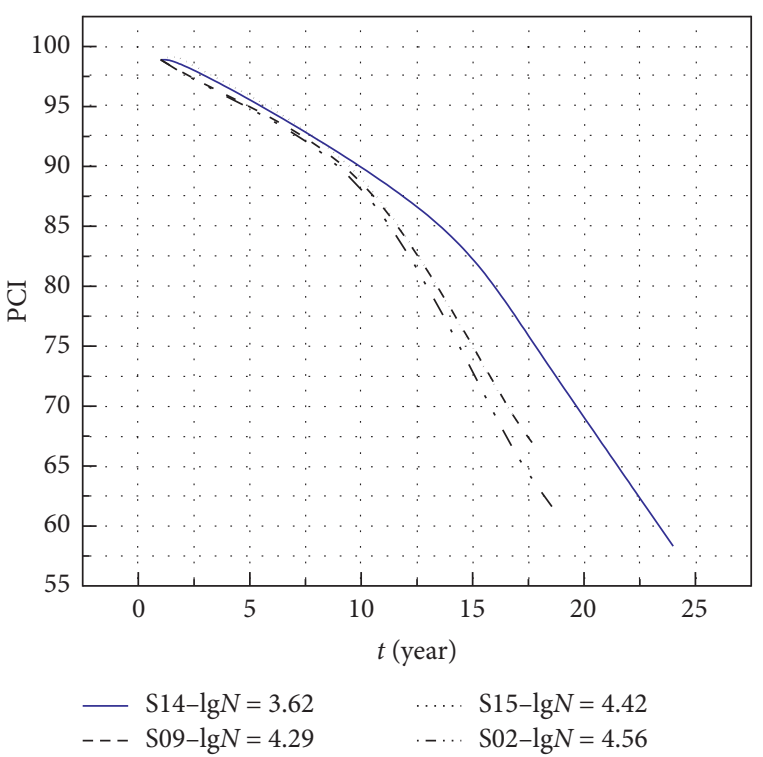

(a)

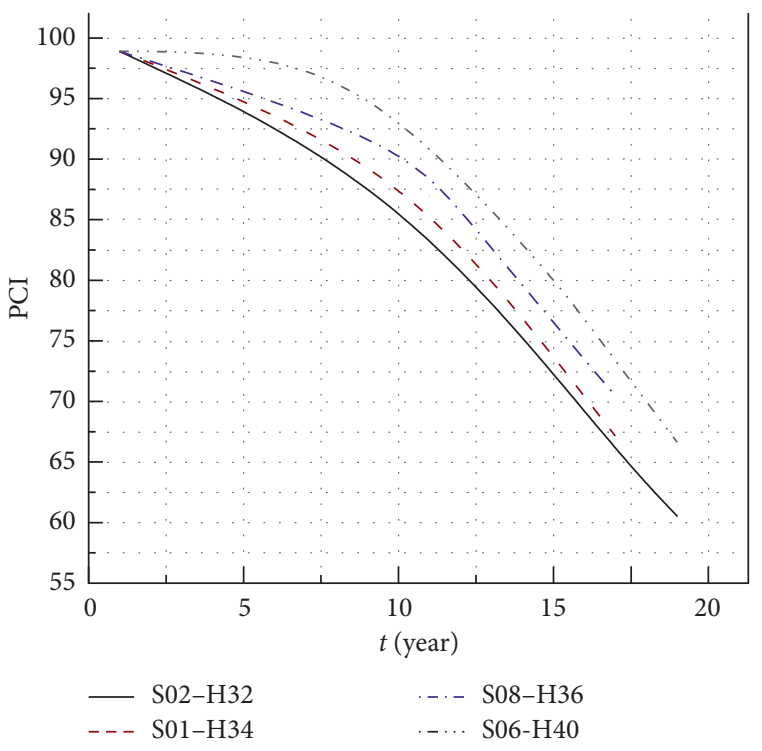

(b)

Figure 4: Model prediction of levels of parameters: (a) different flying traffic volumes $(H=32 \mathrm{~cm})$ and $(\mathrm{b})$ different track surface thickness grades.

following reasons were considered. In this study, the logarithmic conversion of aircraft takeoff and landing frequencies was used to analyze the values, so the logarithmic values of the three levels of traffic had a small pairwise difference $(4.42-4.29=0.13$ and $4.56-4.42=0.14)$. However, the actual traffic volume difference of the three levels was about 6,804 , which was quite different.

This might have indicated that, in the case of a cement concrete surface thickness of $32 \mathrm{~cm}$, the damage trend of the airport pavement performance would change suddenly after the aircraft load times reached a certain quantity level. In other words, for an airport of a certain level, the aircraft traffic volume had an extreme point. When the number of aircraft loads exceeded this extreme point, it might cause relatively significant damage to the airport surface, that is, for an airport with a certain grade index in the flight area (for example, $4 \mathrm{~F}$ or $3 \mathrm{C}$ ) [37], there was an extreme point in the frequencies of aircraft takeoffs and landings. When the amount of aircraft traffic exceeded this extreme point, there might have been significant damage to the airport pavement surface.

6.3.2. Prediction of the Model with Varying Pavement Thicknesses. In order to evaluate the prediction performance of the model in this study for the pavement performance of different pavement thickness levels, the samples S2 $(\lg N=4.56, H=32 \mathrm{~cm}), \mathrm{S} 1 \quad(\lg N=4.56, H=34 \mathrm{~cm}), \mathrm{S} 8$ $(\lg N=4.65, H=36 \mathrm{~cm})$, and S6 $(\lg N=4.65, H=40 \mathrm{~cm})$ with similar aircraft traffic levels were selected from dataset 1 according to the principle of large residuals. The prediction performance curve is shown in Figure 4(b).

It can be seen from Figure 4(b) that the prediction curve of the mixed-effect model could better fit the PCI of the pavement of different pavement thickness levels (when the volume of the aircraft traffic was constant). The PCI degraded curves of different pavement thickness levels were significantly different. As the thickness decreased, the value of the PCI decreased, and the smaller the thickness was, the greater the degraded rate of the PCI was. This result indicated that, with the decrease of the pavement thickness, the pavement structural load-bearing capacity decreased, which led to greater damage for the pavement at the same aircraft traffic level when the PCI value was smaller. This also proved that the mixed-effect model could capture the deterioration trends of individual pavement surfaces with different thickness levels better (limited to the pavement thickness range included in the dataset).

It is worth noting from Figure 4(b) that the remaining life of the corresponding pavement was approximately increased by three years when the pavement thickness increased by $8 \mathrm{~cm}(\mathrm{H} 40-\mathrm{H} 32)$. In the literature [38], it was pointed out that the residual life of the pavement was related to the third power of the pavement thickness, and it was not reasonable to calculate the residual life of cement concrete pavement with the pavement design reversal method. Compared with the literature [38], the sensitivity of the performance model determined by the mixed-effect and joint-estimation method in this study for the residual life of airport pavement was reduced. The estimation result of the residual life was more reasonable. The accuracy still needs further study.

6.3.3. Extrapolation Performance of Random-Effect Mixed Model. In order to further analyze the performance of the mixed-effect model obtained in this study to predict data outside the dataset used, that is, the extrapolation performance of the model, the parameter values estimated with the above nonlinear mixed-effect evaluation model equation (9) 


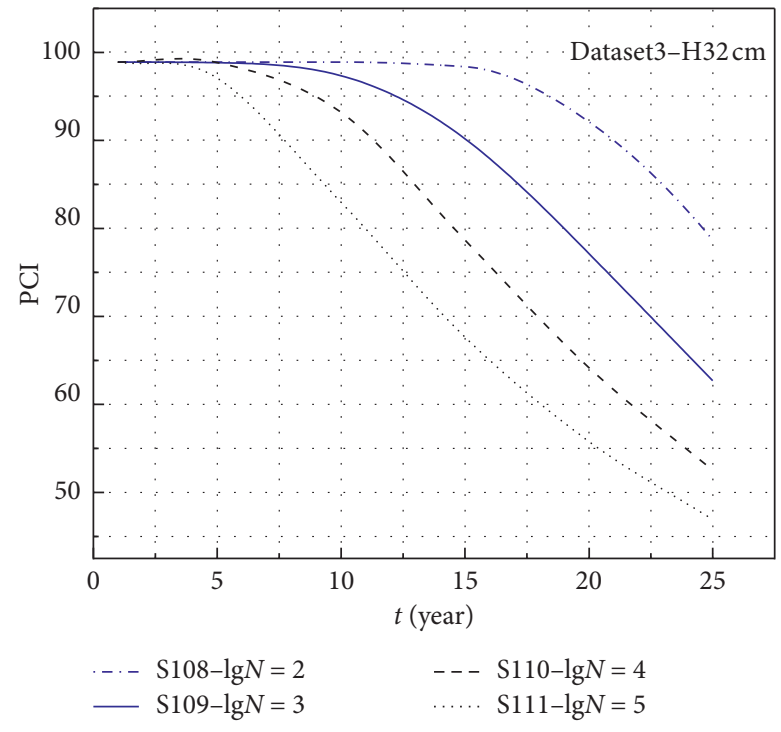

(a)

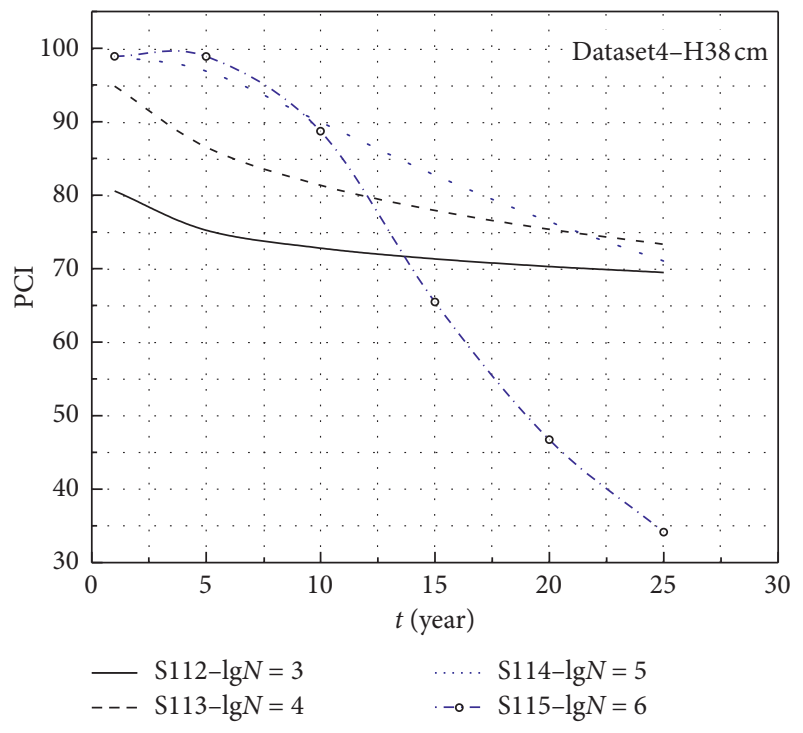

(b)

Figure 5: Analysis of model extrapolation performance for different aircraft traffic levels: (a) dataset 3; (b) dataset 4.

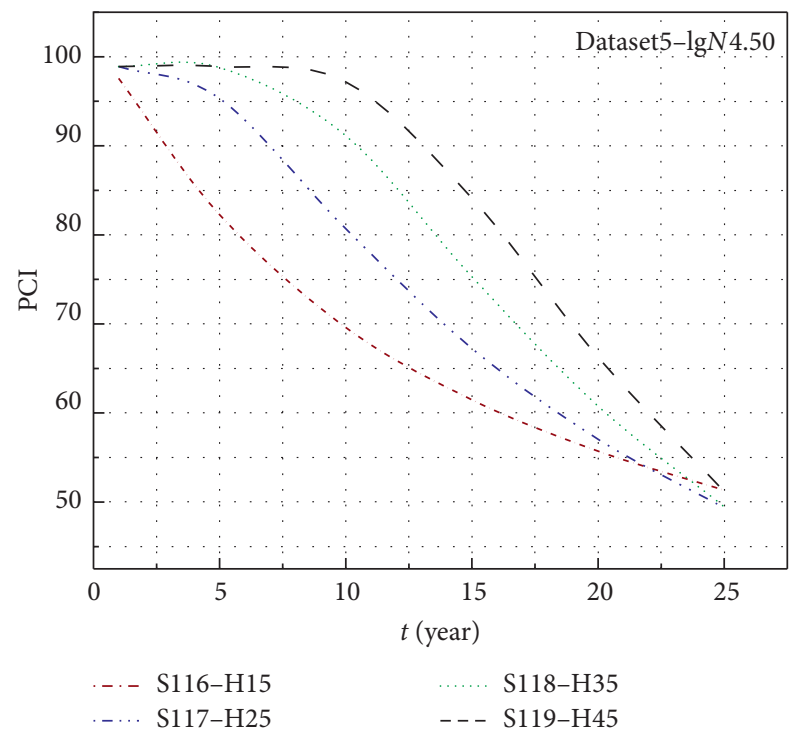

(a)

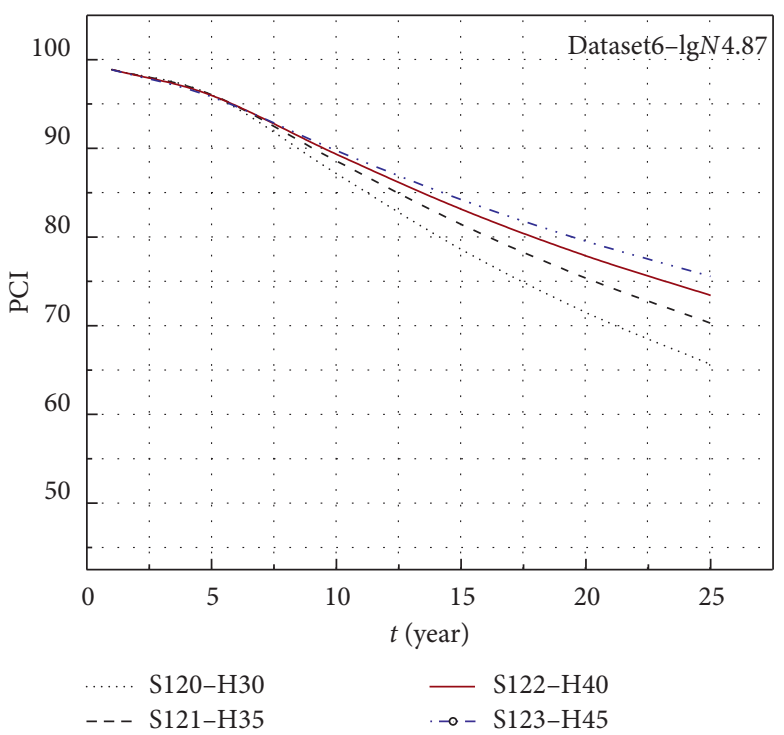

(b)

FIgURE 6: Analysis of model extrapolation performance for different pavement thickness levels: (a) dataset 5; (b) dataset 6.

were used. By setting different levels of aircraft traffic volume and different pavement thickness levels, the mixed-effect model was used to predict the deterioration curve of the PCI of the airport pavement. The results are shown in Figures 5 and 6 .

Taking Figure 5(a) for an example, the data in dataset 3 was used for extrapolation analysis. That is, at the level of pavement surface thickness of $H=32 \mathrm{~cm}$, the PCI values of the samples S108, S109, S110, and S111 with service lives of 1 year, 5 years, 10 years, 15 years, and 25 years were predicted when the $\operatorname{lgN}$ were $2.0,3.0,4.0$, and 5.0 at the aircraft traffic levels, respectively, and the PCI deterioration curve was plotted. Figure 5(b) is similar to Figure 5(a), except that
Figure 5(b) shows the extrapolation capability analysis of dataset 4. In addition, Figure 6 shows similarity to the method shown in Figure 5, except Figure 6 shows the extrapolation capability analysis of different pavement thickness levels at the same aircraft traffic level.

It can be seen from Figure 5(a) that the mixed-effect model using the estimated value of data parameters in dataset $3 \mathrm{had}$ good predictive extrapolation ability for the PCI at different aircraft traffic levels, and the influence characteristic of the aircraft traffic on the PCI was consistent with the conclusions described in the previous section. However, as shown in Figure 5(b), the predictive extrapolation performance of the model was poor and no obvious rules could be obtained. 
For Figures 6(a) and 6(b), the mixed-effect model with the estimated values of the parameters in the two datasets had good extrapolation ability, and the influence characteristic of the pavement thickness on the PCI was consistent with the conclusions described in the previous section.

Based on the comprehensive analysis of the above graphs, the following conclusions could be drawn: the mixed-effect model of airport pavement performance prediction was more sensitive to the pavement surface thickness than the aircraft traffic volume; specifically, the model could better predict the PCI decay peculiarity of different thicknesses of pavements. That is, the model had better predictive extrapolation performance. However, the prediction performance for the aircraft traffic levels was poor, as shown by the fact that the model could better predict the decay peculiarity of the performance of samples contained in the dataset, while the model had poor extrapolation ability for samples outside the dataset (e.g., Figure 5(b) shows that there was no discipline).

In addition, by comparing the above four figures, it could be concluded that the influence of the pavement surface thickness on the prediction performance of the mixed-effect model was greater than that of the aircraft traffic. It is also shown in the figures that the extrapolation capacity of the aircraft traffic volume varied greatly, while the extrapolation capacity of the pavement thickness was relatively significant. These characteristics were not due to a problem with the dataset itself, but rather were determined by the poor extrapolation ability of the mixed-effect model for the data that was not included in the modeling data.

\section{Conclusions}

Through the joint estimation of two datasets, a randomeffect nonlinear mixed model was used to calibrate a functional residual life prediction model for a local civil airport road in Henan Province. A pavement condition index (PCI) prediction model considering the influence of aircraft traffic and the thickness of the pavement surface was proposed. The marginal effect of the validated model was analyzed using actual data. The following conclusions can be derived from the analysis and evaluation of the model.

The joint estimation had the advantages of high identification and significant statistical efficiency. Dataset 1 may have contained unique explanatory variables that were not present in dataset 2 . The joint estimates could identify the effects of these unique variables from the different data sources, thereby determining the parameter estimates for the unique variables. Joint estimation could use all available data (two different datasets 1 and 2) to determine the parameter estimation common to all data sources. Due to the combination of the two data sources, the overall sample size of the model increased, so the variance of the parameter estimation value of the function was smaller, and the statistical efficiency was significant.

Through the analysis of the relationship between the standardized residuals and the predicted value of two different datasets adopted in the model calibration, the results showed that the normality assumption of the residuals of the prediction model established by the random-effect method combined with the joint-estimation approach was easier to satisfy than the nonlinear least-squares method. This also indicated that the statistical results of the random-effect nonlinear mixed model were more reliable, and the fitting effect of the model was significant.

According to the parameter estimation results of the random-effect nonlinear mixed models, using variable difference analysis combined with the prediction performance curve graphic method, the decay rules of the PCI were analyzed for the different aircraft traffic levels and the different thicknesses of the cement concrete pavement surface layers. The prediction performance of the random-effect nonlinear mixed model was illustrated with the graphical method. The mixed-effect model of the airport pavement performance prediction was more sensitive to pavement surface thickness than the aircraft traffic volume was. However, the prediction performance for aircraft traffic levels was poor. The model could better predict the decay peculiarity of the performance of the samples contained in the dataset while the model had poor extrapolation ability for samples outside the dataset. In addition, the influence of pavement surface thickness on the prediction performance of the mixed-effect model was greater than that of the aircraft traffic. The extrapolation capacity of the aircraft traffic volume varied greatly while the extrapolation capacity of pavement thickness was relatively significant.

\section{Data Availability}

The figure and table data used to support the findings of this study are included in the article. In addition, the statistical data and the statistical models are available from the corresponding author upon request.

\section{Conflicts of Interest}

The authors declare that there are no conflicts of interest regarding the publication of this paper.

\section{Acknowledgments}

The authors thank LetPub (http://www.letpub.com) for its linguistic assistance during the preparation of this manuscript. This research was funded by the Technology Research and Development Project of China Communications Construction Company (no. 2017-ZJKJ-PTJS03), National Key Research and Development Program of China (no. 2016YFC0802203-5), Key Research Program in Universities of Henan Province (no. 21B580008), and Science and Technology Project of Henan Province (no. 182102310747), for which the authors are grateful.

\section{References}

[1] F. Liu, M. Q. Sun, and Y. J. Wang, "Research on health monitoring system of airport pavement based on gis and fem," Journal of Highway and Transportation Research and Development, vol. 19, no. 3, pp. 16-19, 2014.

[2] S. D. Kohn, "Development of a pavement maintenance management system. Volume II. airfield pavement distress 
identification manual," Concrete Pavements, vol. 101, no. 1, pp. 453-457, 1976.

[3] M. Y. Shahin, Pavement Management for Airports, Roads, and Parking Lots, Springer, New York, NY, USA, 2005.

[4] US Department of Transportation, Federal Aviation Administration, Research Directorate for Airport Technology, and Airport Technology Branch, Airport Pavements-Solutions for Tomorrow's Aircraft, FAA Technical Center New Jersey, Egg Harbor Township, NJ, USA, 1993, https://www.airporttech.tc. faa.gov/Products/Airport-Pavement-Papers-Publications.

[5] J. M. Ling, J. Yuan, and S. B. Xi, "Development of shanghai airport pavement management system," Journal of Tongji University, vol. 33, no. 8, pp. 1041-1046, 2005.

[6] MH/T 5024-2009, Technical Specification of Aerodrome Pavement Evaluation and Management (Chinese Standard), Communication Press Co. Ltd., Beijing, China, 2009.

[7] E. Y. J. Chou, H. Pulugurta, and D. Datta, Pavement Forecasting Models. FHWA/OH-2008/3, pp. 20-28, Department of Transportation, Columbus, OH, USA, 2008.

[8] M. Shahin and J. Walther, Pavement Maintenance Management for Roads and Streets Using the Paver System. M90/05, US Army Construction Engineering Research Laboratory, Washington, DC, USA, 1990.

[9] Federal Aviation Administration, Airport Pavement Design and Evaluation Advisory Circular AC 150/5320-6F, USA Department of Transportation, Washington, DC, USA, 2016.

[10] Federal Aviation Administration, Airport Pavement Design for the Boeing-777 Airplane dated Advisory Circular 150/5320-16, USA Department of Transportation, Washington, DC, USA, 1995.

[11] J. M. Ling and Y. F. Zheng, "Prediction method of remaining life for cement concrete pavement of airport," Journal of Tongji University, vol. 29, no. 4, pp. 484-488, 2001.

[12] R. L. Lytton, "Concepts of pavement performance prediction and modeling," in Proceedings of the Second North American Conference on Managing Pavements, Toronto, Canada, November 1987.

[13] F. Hong and J. A. Prozzi, "Estimation of pavement performance deterioration using bayesian approach," Journal of Infrastructure Systems, vol. 12, no. 2, pp. 77-86, 2006.

[14] M. Black, A. T. Brint, and J. R. Brailsford, "A semi-Markov approach for modelling asset deterioration," Journal of the Operational Research Society, vol. 56, no. 11, pp. 1241-1249, 2005.

[15] K. Golabi, R. B. Kulkarni, and G. B. Way, "A statewide pavement management system," Interfaces, vol. 12, no. 6, pp. 5-21, 1982.

[16] A. A. Butt, M. Y. Shahin, K. J. Feighan, and S. H. Carpenter, "Pavement performance prediction model using the Markov process," in Transportation Research Record (1123), Transportation Research Boardno. 1123, Washington, DC, USA, 1987.

[17] K. A. Abaza, "Empirical approach for estimating the pavement transition probabilities used in non-homogenous Markov chains," International Journal of Pavement Engineering, vol. 18, no. 2, pp. 128-137, 2017.

[18] J. J. Ortiz-García, S. B. Costello, and M. S. Snaith, "Derivation of transition probability matrices for pavement deterioration modeling," Journal of Transportation Engineering, vol. 132, no. 2, pp. 141-161, 2006.

[19] E. V. Ana and W. Bauwens, "Modeling the structural deterioration of urban drainage pipes: the state-of-the-art in statistical methods," Urban Water Journal, vol. 7, no. 1, pp. 47-59, 2010.

[20] K. A. Abaza, "Back-calculation of transition probabilities for Markovian-based pavement performance prediction models," International Journal of Pavement Engineering, vol. 17, no. 3-4, pp. 253-264, 2016.

[21] L. E. Amador-Jiménez and D. Mrawira, "Reliability-based initial pavement performance deterioration modelling," International Journal of Pavement Engineering, vol. 12, no. 2, pp. 177-186, 2011.

[22] K. A. Abaza and M. M. Murad, "Dynamic probabilistic approach for long-term pavement restoration program with added user cost," Transportation Research Record, vol. 1990, no. 1, pp. 48-56, 2007.

[23] X. J. Liu and Y. J. Zheng, "Research on multi-index forecasting method of asphalt concrete pavement performance based on grey theory," Highway, vol. 57, no. 4, pp. 233-237, 2012.

[24] K. Z. Yan, J. L. Wu, and J. Z. Zhang, "Performance evaluation of asphalt pavement based on multi-level grey theory," Bulletin of Science and Technology, vol. 1, pp. 98-102, 2009.

[25] G. X. Wang, J. F. An, and R. S. Chen, "Application of grey theory in pavement performance prediction," Journal of Highway and Transportation Research and Development, vol. 19, no. 3, pp. 16-19, 2002.

[26] M. Ben-Akiva and R. Ramaswamy, "An approach for predicting latent infrastructure facility deterioration," Transportation Science, vol. 27, no. 2, pp. 174-193, 1993.

[27] K. Ashok and M. E. Ben-Akiva, "Alternative approaches for real-time estimation and prediction of time-dependent origin destination flows," Transportation Science, vol. 34, no. 1, pp. 21-36, 2000.

[28] Z. Lou, J. J. Lu, M. Gunaratne, and B. Dietrich, "Forecasting of pavement crack performance with adaptive filter model," Transportation Research Record, vol. 1699, no. 1, pp. 95-100, 2000.

[29] J. A. Pinheiro and D. M. Bates, Mixed-effects Models in S and S-Plus, Springer, New York, NY, USA, 2000.

[30] J. C. Wang, H. Y. Xie, and B. F. Jiang, Multi-level Statistical Analysis Model-Methods and Applications, Higher Education Press, Beijing, China, 2008.

[31] A. R. Archilla, P. S. Ooi, and K. G. Sandefur, "Estimation of a resilient modulus model for cohesive soils using joint estimation and mixed effects," Journal of Geotechnical and Geoenvironmental Engineering, vol. 133, no. 8, pp. 984-994, 2007.

[32] T. Morikawa, M. Ben-Akiva, and K. Yamada, "Forecasting intercity rail ridership using revealed preference and preference data," Transportation Research Record, vol. 1328, pp. 30-35, 1991.

[33] A. R. Archilla and S. Madanat, "Estimation of rutting models by combining data from different sources," Journal of Transportation Engineering, vol. 127, no. 5, pp. 379-389, 2001.

[34] J. A. Prozzi and S. M. Madanat, "Development of pavement performance models by combining experimental and field data," Journal of Infrastructure Systems, vol. 10, no. 1, pp. 9-22, 2004.

[35] J. Yuan and E. H. Su, "Performance Pavement prediction based on linear mixed effects model," Journal of Tongji University (Natural Science), vol. 42, no. 5, pp. 123-135, 2012.

[36] L. J. Sun, Structural Behaviour of Asphalt Pavement, Tongji University Press, Shanghai, China, 2013.

[37] MH 5001-2013, Aerodrome Technical Standards (Chinese Standard), Communication Press Co., Ltd., Beijing, China, 2013.

[38] G. Yang, "Research on evaluation and prediction of residual life of cement concrete pavement of airport in cold region," Master's thesis, Harbin Institute of Technology, Harbin, China, 2017. 